\title{
Publisher's Note: Band bowing and the direct-to-indirect crossover in random BAIN alloys [Phys. Rev. Materials 1, 065001 (2017)]
}

\author{
Jimmy-Xuan Shen, Darshana Wickramaratne, and Chris G. Van de Walle
}

(Q) (Received 27 December 2017; published 3 January 2018)

DOI: 10.1103/PhysRevMaterials.2.019901

This paper was published online on 13 November 2017 with an error in the Acknowledgments. On page 6, the first sentence of the Acknowledgments should read as "J.S. was supported by the National Science Foundation (NSF) under Grant No. DMR-1434854." The Acknowledgments have been corrected as of 22 December 2017. 